gedrungen sind; siehe z. B. 22, 19. 103, 19/20. 104, 15. 108, 3. 111, 14 . 112, 19. 117,1 . 119,22 . 135,8 u. 18. 141,9 . $148,3 / 4$.

Eine Überarbeitung, die den alten Text in $\mathbf{v}$ verdrängt hat, ist in gleicher Weise neben der richtigen Lesart in T 164, 20/21 eingedrungen und dadurch als sehr alt erwiesen.

Außer den alten Fehlern und Überarbeitungen findet sich in $\mathrm{T}$ noch eine Fülle specieller Lesarten, die sich am wahrscheinlichsten so erklären, daß sie im Lauf der besondern Überlieferung dieser Handschrift entstanden sind. Sie begegnen auf jeder Seite des kritischen Apparats, und es ist überflüssig, hier Beispiele anzuführen.

Ich habe hier etwas den Stab über den Codex T gebrochen, aber um ihn gerecht zu beurteilen, darf man die zahlreichen Fälle nicht außer Ansatz lassen, wo er sich den besten Handschriften an die Seite stellt und uns so große Dienste leistet.

\title{
VII. Cassiodor
}

Der außerordentliche Wert, den die Übersetzung des Cassiodor ${ }^{1}$ ) für die Arbeit der Auswahl unter den Lesarten besitzt, ist schon oben hervorgetreten. Cassiodor darf mit B, dem seine Quelle nahe stand (s. S. XXXVIf), als einer der treusten Vertreter der Überlieferung betrachtet werden.

Ich habe bereits (S. XLIX) festgestellt, daß da, wo er Theodorus selber übersetzt, sein Text im allgemeinen von den Entstellungen unsrer Handschrift der Tripartita frei geblieben ist. Sobald er seiner eignen Handschrift von Theodoret folgt, stellt er immer eine bessere Überlieferung als T dar. Es genügt z. B. ein Blick auf die ganze lange Urkunde II 8, um zu sehen, daß die Überlieferung Cassiodors vortrefflich ist, und die Kontrolle seiner Handschriften hat mir zuweilen ermöglicht, sie noch zu verbessern; z. B. 114, 5. Da boten mir die Ausgaben von Cass. omnibus $=\pi \tilde{\alpha} \sigma \iota \nu$, Fehler von $\mathrm{T}$. Aber in seinen besten Handschriften (LP) fand ich omnia omnibus $=\pi \alpha \nu \tau \alpha \dot{\alpha} \alpha \sigma \iota \nu$ Brv, wodurch die peinliche Treue des Übersetzers bestätigt wird. Ich habe den Eindruck, daß, gerade umgekehrt wie Theodorus Lector, Cassiodor niemals, wenigstens soweit es Theodoret betrifft, den Text seiner griechischen Handschrift mit Vorbedacht umgearbeitet oder gefälscht hat; s. in dieser Hinsicht seine mit B und $V^{2}$ gemeinsamen Fehler (S. XXXVII u. XL).

1) Ich habe durchweg den Namen Cassiodor und nicht Epiphanius gebraucht, unter anderm deshalb, weil der letztere Name (s. S. $25 \mathrm{ff}$ ) für einen andern Epiphanius aufgespart werden sollte. 
Ebenso legen manche Übersetztngsschnitzer Zeugnis von seiner

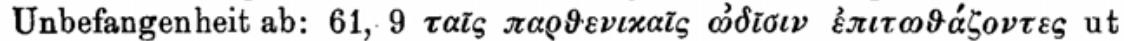
illic virginum cantibus insultarent. 168,9 xà̀ $\pi 0 \lambda \lambda \grave{\alpha} \varsigma \mu \grave{\varepsilon} \nu$ $\xi \lambda \varepsilon \pi \lambda^{\lambda} \varepsilon \iota \varsigma \tau \tilde{\varphi} \tau \varepsilon i \chi \varepsilon \iota \pi \varrho o \sigma \varepsilon v \varepsilon \gamma \chi \omega^{\prime}$ et multas quidem cepisset civitates. 264, 6 ff oíóv $\tau \iota \varsigma$ $\pi \alpha \iota \delta o \tau \varrho i \beta \eta \varsigma$ velut puer exercitatus usw.

Die Beispiele ließen sich noch vermehren. Das Wesentliche ist, daß der Übersetzer sich immer ausschließlich an seine Handscbrift hält, indem er nichts sonst zu Rate zieht und so wenig wie möglich seine Einbildungskraft wirken läßt. S. in dieser Beziehung 9,13/14 omnes ... vocem (so Codd.CLP!) Cass., wo vielleicht die Spur eines Fehlers einer alten Quelle erhalten ist; vgl. ebd. die Lesarten von An. - 265, $9 \pi 0 \tilde{v}$ $\sigma \grave{v} \beta \alpha \delta i \zeta \varepsilon \iota$ s. Theodoret hat in dieser Frage sicher in familiärem Ton $\pi o \tilde{v}$ (und nicht $\pi \circ \tau \mathrm{FGy}$ ) geschrieben. Cassiodor bildet getreu nach: ubi vadis.

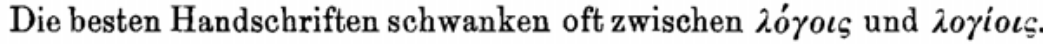
Cass. liefert einen sichern Prüfstein, weil er immer sorgfältig unter-

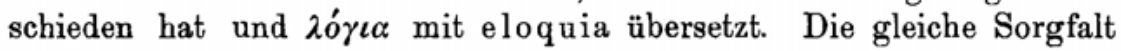
verwendet er auf die treue Wiedergabe der Tempora beim Verb, der Partikeln und der Wortstellung. Endlich sind selbst die Auslassungen von Cass. lehrreich, weil sie oft bezeugen, daß er schon einen unübersetzbaren Text vorfand $(106,23.257,17 / 18.272,4 / 5)$.

Es scheint mir also, daß Cassiodor an seinem Texte nur das Minimum von Änderungen vorgenommen hat, das. für einen Übersetzer unvermeidlich ist, falls ihm daran liegt, seine Vorlage gewissenhaft nach seinem Können und seinen Kräften in andrer Sprache wiederzugeben. Trotz ihrer Irrtümer und Widersinnigkeiten darf man den Wert seiner Arbeit nicht herabdrücken. Es war nicht leicht, die oft spitzfindigen, dunkeln und schlecht erhaltenen Urkunden wie I 4, II 8 und noch viele andre ins Lateinische zu übertragen, und Cass. ist damit auf eine im allgemeinen erträgliche Art fertig geworden.

Nicht als Überarbeitungen im eigentlichen Sinne betrachte ich die Fälle, wo Cassiodor eine Wendung oder ein Tempus hat ändern oder auch ein Wort hat bessern oder hinzufügen müssen, um den Satz, den er in seinem Texte vorfand, annähernd verständlich zu machen. In gleichen Fällen würde wohl noch heute ein Übersetzer, der seiner Aufgabe gewachsen ist, zu ähnlichen Mitteln greifen. Beispiele für derartige Emendationen s. oben S. XXX gelegentlich der Übereinstimmungen von $\mathrm{H}$ mit Cass. Zum Beispiel Cass. hat auch 147, 11, um einen Satz zu gewinnen, der einigermaßen Sinn gibt, hinzugefügt quae gesta sunt, und wohl im Anschluß an ihn oder mindestens aus

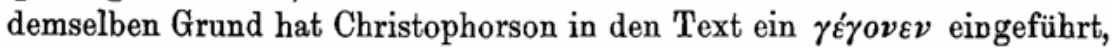


das alle späteren Ausgaben beibehalten haben. Aber die Verderbnis geht in die Zeit vor unserm Archetypus zurück.

Wenn Cassiodor nur kurze Excerpte vornimmt und sie in den Text eines andern Autors einschiebt, kann er zuweilen nicht umhin, zu kürzen, umzuarbeiten und überhaupt etwas freier $\mathrm{za}$ schreiben. Aber solche Fälle sind für Theodoret selten.

Gewiß gibt es in unserm Text der lateinischen Tripartita Entstellungen und Überarbeitungen - welche Handschrift ist davon ganz frei? -, aber ich glaube, die Änderungen gehen selten auf Cassiodor zurück, und selbst die später eingedrungenen haben keine große Bedeutung und niemals den tendenziösen Charakter, der uns in der griechischen Tripartita entgegengetreten ist.

In dem nach der Tripartita übersetzten Teil von Cass. verdient nur ein etwas charakteristischer Zusatz hervorgehoben zu werden: 8, 22. Er kann später sein als Cassiodor.

Im allgemeinen darf man daraus, daß Cass. ein Wort mehr als der Grieche oder sonst eine Abweichung enthält, nicht schließen, daß an der Stelle eine absichtliche Änderung seinerseits vorliege:

$61,8 \dot{\alpha} x o \lambda \dot{\alpha} \sigma \tau o v$ daípovos + id est Veneris Cass. Sicher hat die Feder Theodorets sich gesträubt den Namen der Göttin der Begierde (vgl. 201, 10) zu schreiben, den er jedoch in seiner Quelle Euseb. III 26 vorfand. Aber die Glosse 'A drei Überarbeitungen, hinzugefügt, und Cass. hat sie wohl bereits am Rande seiner Handschrift gefunden.

68, $1 \pi \varrho 0 \sigma \tilde{\eta} x \varepsilon \varphi \varrho \alpha ́ \sigma \alpha \iota]$ loqui decet + audite Cass., aber ebenso +

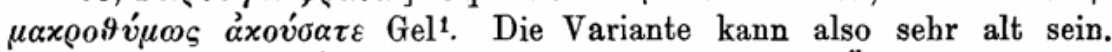

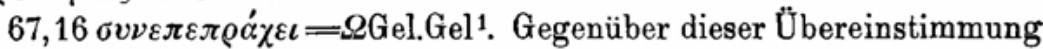
hätte ich nicht zu versichern gewagt, daß confudisset Cass. (= conturbasset $\Lambda$ ) für die Quelle des Theodoret die Form $\sigma v v \varepsilon \tau \varepsilon \tau \alpha \rho \alpha \dot{\alpha} \chi \varepsilon \iota$ sicher stellte, die Balforeus aufnahm.

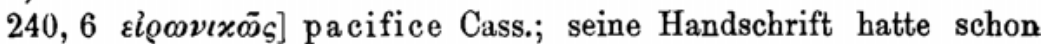

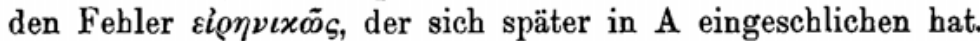

Anderwärts hat Cass. offenbar allein die richtige Lesart erhalten, z. B. $67,1.135,18$; vgl. auch ${ }^{\prime}$ (?) $141,12.250,15.332,2$.

\section{SchluB folgerungen}

Aus unsrer ganzen Untersuchung ergibt sich, daß die Überlieferung im allgemeinen erlaubt, den Text des Theodoret auf Grundlagen von bemerkenswerter Sicherheit aufzubauen. Mit Handschriften wie A, n, B, $V^{2}$, bei besonnener Verwertung der syrischen Bruchstücke, der Tri- 